\title{
Hybrid Energy Storage Module Utilizing Hardware-in-the-Loop Emulated Distributed Generation
}

\author{
Jacob L. Sanchez, Brian. J. McRee, David A. Wetz ${ }^{*}$ \\ Electrical Engineering Dept., The University of Texas at Arlington, 416 Yates St, Arlington, TX 76019, USA.
}

\begin{abstract}
How to cite this paper: Jacob L. Sanchez, Brian. J. McRee, David A. Wetz. (2021) Hybrid Energy Storage Module Utilizing Hardware-in-the-Loop Emulated Distributed Generation. Journal of Electrical Power \& Energy Systems, 5(1), 46-57. DOI: $10.26855 /$ jepes.2021.05.002
\end{abstract}

Received: April 7, 2021

Accepted: April 30, 2021

Published: May 12, 2021

*Corresponding author: David A. Wetz, Electrical Engineering Dept., The University of Texas at Arlington, 416 Yates St, Arlington, TX 76019, USA.

Email: wetz@uta.edu

\begin{abstract}
Future electrical power systems will integrate a significant number of distributed generation sources that are all intelligently monitored and controlled. It is not always possible to have each potential type of generation source present for laboratory evaluation, and thus hardware-in-the-loop has emerged to utilize hardware validated models as hardware emulated sources in laboratory testbeds. At the University of Texas at Arlington, a low power testbed has been setup on which real and emulated generation sources can be seamlessly integrated and studied under load profiles representative of an end user's application. In the work presented here, a hybrid power system that integrates batteries, ultracapacitors, and an AC generator is being studied. The AC generator is implemented using hardware and then emulated using an OPAL-RT hardware-in-the-loop platform. The validity of using hardware-in-the-loop to emulate these types of sources as well as the ability of the hardware energy storage to buffer the generator is being studied. A description of the hardware setup will be presented along with results collected to date.
\end{abstract}

\section{Keywords}

Power Quality, Batteries, AC Generators

\section{Introduction}

Previous work [1-5] performed at the Pulsed Power and Energy Lab (PPEL) at The University of Texas at Arlington (UTA) has shown the efficacy of Hybrid Energy Storage Modules (HESM) in buffering a rotating machine while transient loads are sourced. In Cohen, D. A. Wetz, C. Storm, and J. Heinzel [4], the rotating machine was a single-phase gasoline generator fed into a commercially available DC power supply. In the work presented here, the gasoline generator has been replaced with a 3-phase electric motor-generator pair. Experiments have been performed in which the motor-generator is physically present and also in the scenario where the same motor-generator source is emulated using a hardware in the loop platform (HIL). While there is existing work discussing the emulation of power hardware with HIL systems [6-9]. The goal of this work is to demonstrate the validity of studying a general power system using sources that are emulated using HIL by comparison to real hardware testbeds. While some basic comparisons between the real and emulated generators, respectively, will be given, further work towards 
more fully modeling the motor-generator is necessary. This paper does not expand or discuss the theoretical backing of PHIL in detail. The scope of this paper is to present work completed thus far as an intermediate step in the development of fully validated PHIL integration for expansion to a medium voltage testbed.

\section{Background}

A HESM has been previously shown to be able to buffer the power draw of a conventional gasoline powered generator [1]. A HESM generally consists of a combination of high energy density devices and high-power density devices [10]. The energy dense devices can store a large amount of energy, but they typically have a lower peak power output, such as lithium-ion batteries and flywheels. These devices are intelligently controlled to deliver a portion of the energy needed by the pulsed load and the rest of the needed power is supplemented by the prime source. The power dense devices can deliver high peak power, but typically lacks in the magnitude of energy that can be stored, such as electric double layer capacitors (EDLCs) for example. These devices can quickly deliver the high-power transient portions of the pulsed load while the prime power and energy dense sources, respectively, ramp up to full load. When buffered by a HESM properly, the generator can provide constant and efficient power, while the HESM's battery/EDLC is able to supply or sink, respectively, excess transient power. Figure 1 graphically demonstrates an actively controlled energy dense battery HESM with a floating power dense EDLC. Previous work [1] at UTA involved constructing a HESM using commercial off the shelf (COTS) components, pictured in Figure 2.

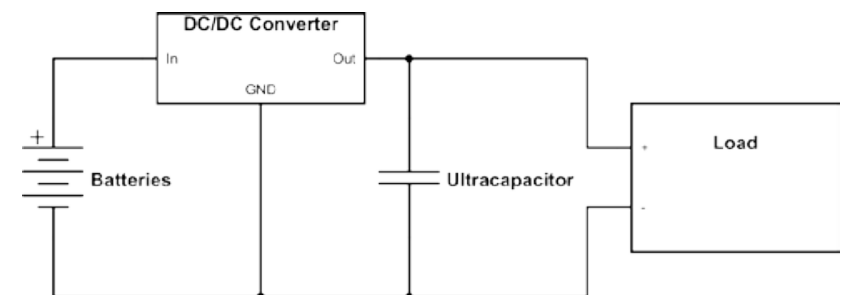

Figure 1. Simplified schematic of an active HESM utilizing a passive EDLC.

Power electronic buck and boost converters, respectively used for power regulation

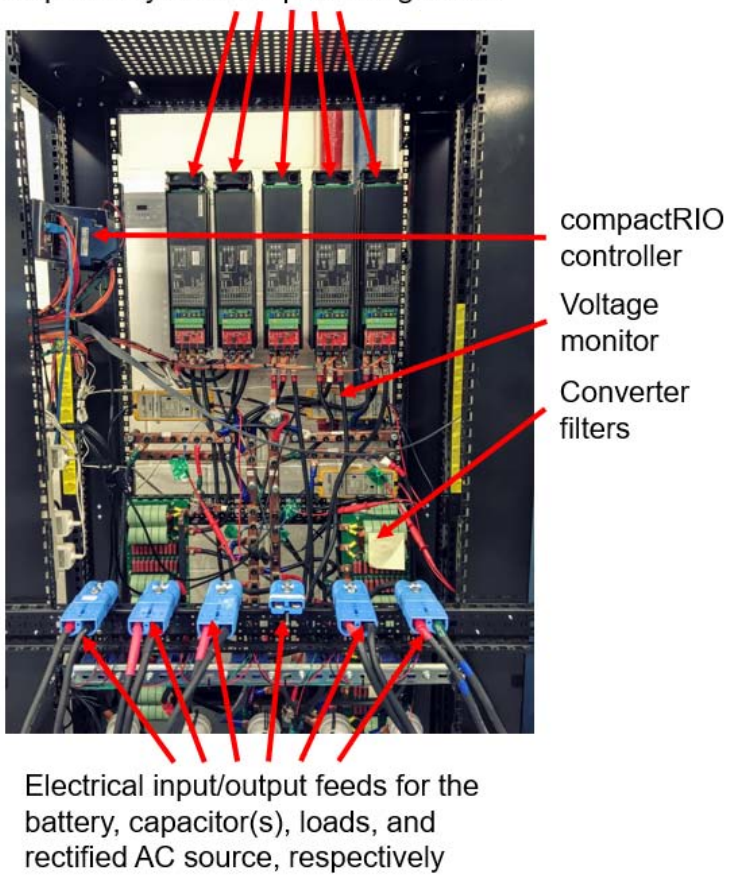

Figure 2. UTA Pulsed Power and Energy Lab’s (PPELs) COTS HESM (Battery, EDLCs, HIL platform, power supply, and AC loads not shown here). 
This COTS HESM was used as the HESM for buffering the motor-generator in this work. Power Hardware in the Loop (PHIL) offers potential benefits to the design and development process of power systems [6-8]. PHIL allows for the real-time emulation of a physical component or control system that is not in possession [9]. As seen in Figure 3 , a model of the desired component is deployed on a real-time computer system. The HIL simulator provides analog outputs that are connected to hardware amplifiers that can emulate the component in the experiment being performed. In real-time, measurements taken from the experiment can be fed back to the HIL simulator where the model is adjusted, and the analog outputs are changed accordingly. In the experiment being performed here, this allows for an AC power supply to mimic the behavior, power quality, etc. of a motor-generator set that is not in possession. This means that the behavior of the system can be well studied without procuring the device itself. This validity of the emulated power component is, of course, limited to the validity of the model used. However, despite the need for rigorous model validation, system level integration of emulated components can be achieved rapidly. UTA possesses an OPAL-RT real-time simulator, also pictured in Figure 3, which is being used in the work presented here.

\section{Motor-Generator Simulation}

The OPAL-RT HIL simulator utilizes MATLAB Simulink ${ }^{\circledR}$ as the software platform on which models are developed and deployed. In this work, a simple model of a motor-generator pair was developed using electrical and mechanical parameters from the hardware motor-generator. It was actualized using existing blocks in the Simulink Power Systems toolbox in MATLAB Simulink, shown in Figure 4.

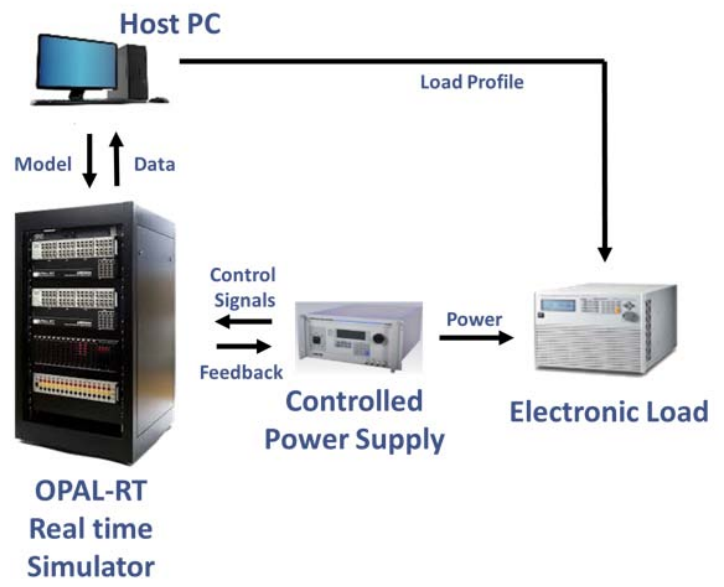

Figure 3. Power systems are emulated by a high slew rate power supply (Controller Power Supply) modulated by a real-time model in the OPAL-RT simulator. This feed forward control and measured feedback allows for a simulation model to interact and be affected by real hardware (electronic load).

The model in Figure 4 is a very simple model of a $2 \mathrm{~kW}$ induction motor/synchronous generator set, which is possessed by UTA. The mathematical model of the motor-generator set is the one that is pre-built into the Simulink toolbox. The motor-generator parameters are filled in using the manufacturer's datasheets and independent validation experiments, which will not be discussed in detail here, were used to tweak those for better results. The validation experiments performed involved step loading the generator under loads ranging from $100 \mathrm{~W}$ up to $2 \mathrm{~kW}$ and running the generator under continuous loads across the same range. The experimental data was compared with simulation results into a similar load to find parameters that provided the most consistent results across the range.

There is of course no perfect solution since there are many non-ideal elements within the entire setup that are not considered but the most feasible solution was found and used. It should be emphasized here that the intent of this work is to quickly study the feasibility of using this type of simple model, with minimal model validation, to obtain results that are like those obtained with hardware when deployed using PHIL. If the results are promising, then it provides insight for how much effort is needed to develop models of more relevant motor-generator sets. The ability to emulate hardware in this manner is incredibly beneficial since it is always difficult and costly to procure large scale hardware for laboratory testing. 
Once the model is running, the generator's stator voltages are output to a high slew-rate 3-phase power supply. During tests, the loaded line currents of the power supply are measured and fed back into the OPAL-RT. Based on these measurements, the stator voltages and generator frequency are adjusted in the model. On the next time step, adjustments are made to the OPAL-RT's analog outputs, which in turn alter the 3-phase voltages of the power supply. This process repeats for the duration of the experiment allowing for the emulation of the physical motor-generator set.

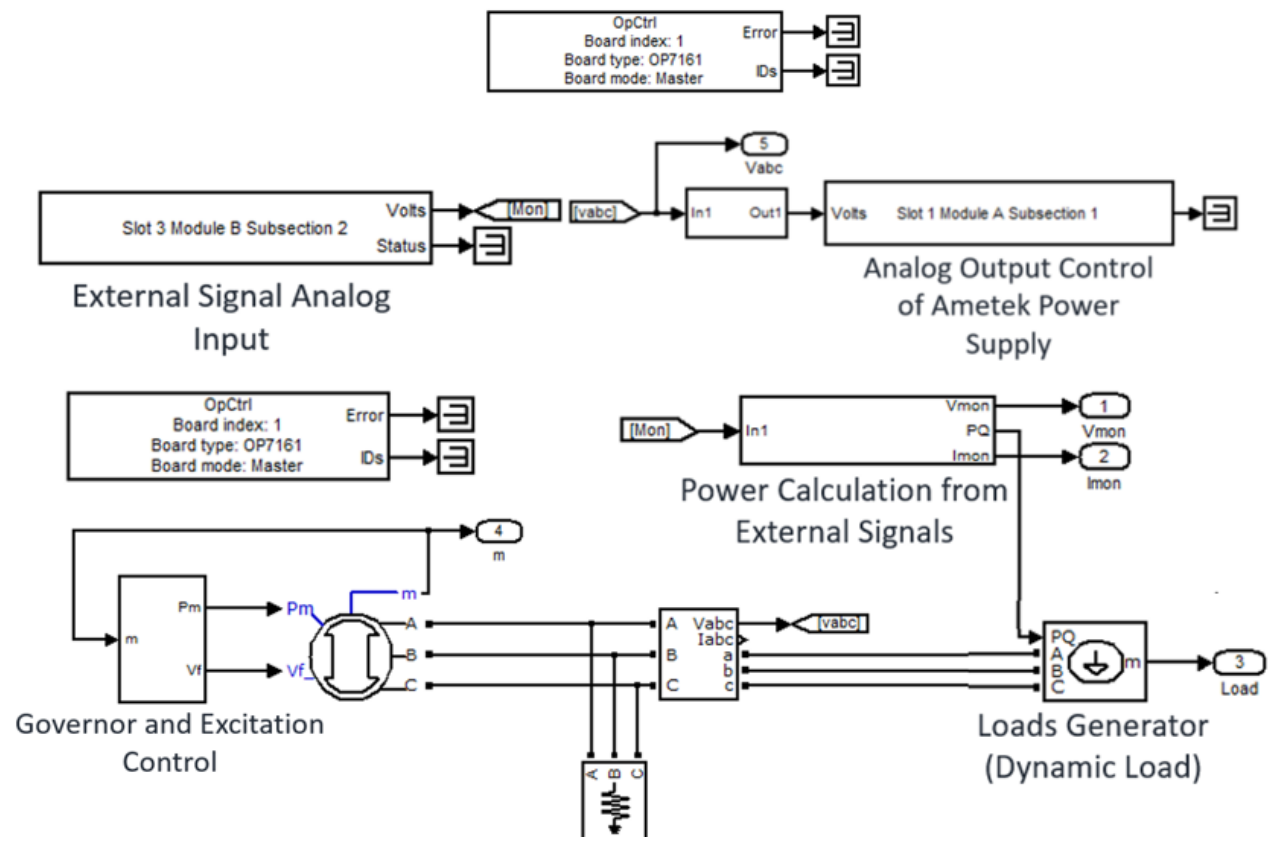

Figure 4. Simulink ${ }^{\circledR}$ block diagram used to emulate a motor-generator set in real-time using the OPAL-RT real-time simulator.

\section{Experimental Setup}

Figure 5 shows a conceptual block diagram of the hardware setup being used. Starting from the left, a 3-phase AC source, implemented using either hardware or through PHIL emulation, feeds a step-down transformer at roughly 208 VAC. Moving to the right, the transformer steps down the voltage from 208 VAC to 20.8 VAC. Next, the 3-phase waveform is rectified to roughly 30 VDC using an SCR-based 3-phase rectifier, commonly known as a 6-pulse rectifier. The rectified DC voltage is regulated using a DC/DC converter and that converter's output voltage is connected to a DC bus that serves as a point of common (PCC) coupling with the HESM. The PCC is loaded using a DC programmable load, which in this case is utilized in a constant current mode. The HESM contains the energy dense batteries and power dense ultracapacitors that are utilized to buffer the 3-phase source.

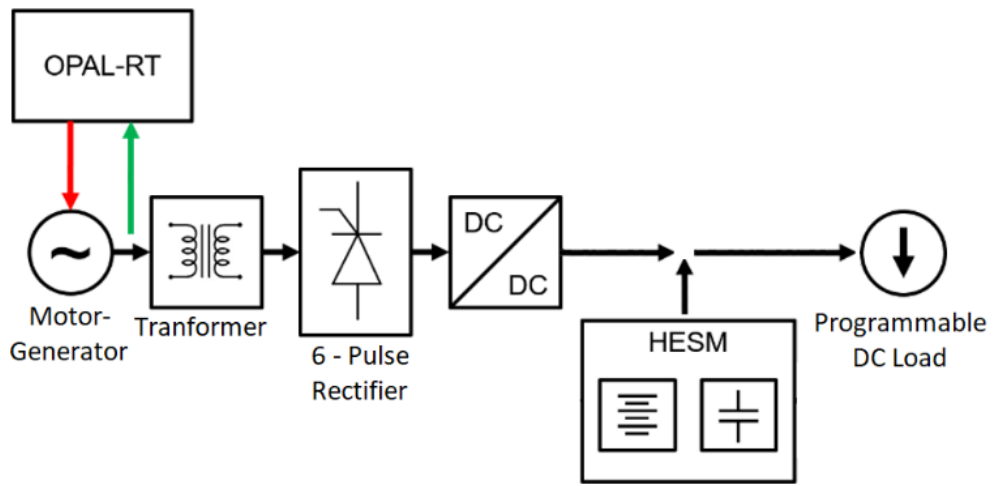

Figure 5. Conceptual block diagram of the experimental setup. 
The motor-generator pair was procured from Festo Didactic, shown in Figure 6. The motor is a LabVolt series 8505 3-phase wound-rotor induction motor. The generator is a LabVolt series 8507 3-phase synchronous generator. Paired together, they allow for a maximum power output of around $1 \mathrm{kVA}$. Both are rated for a stator voltage of 208 VAC at $60 \mathrm{~Hz}$.
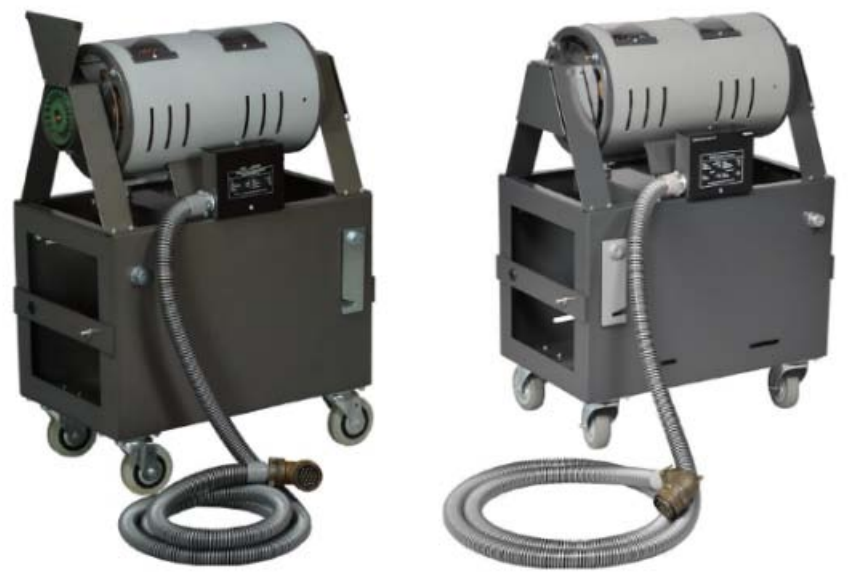

Figure 6. 3-phase induction motor (left) and 3-phase synchronous motor/generator (right). Both are rated at 208 VAC, 2 kVA.

The power supply that is used to amplify the OPAL-RT HIL system is a California Instruments CSW 5550,

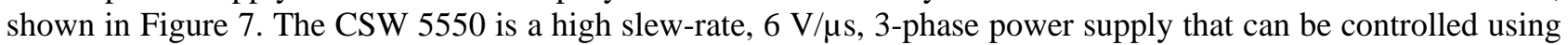
analog reference signals input to the supply. These analog inputs allow the power supply to emulate the individual phase voltages of a real-world generator.

Figure 8 depicts a more detailed diagram of the experimental setup utilizing the motor-generator. The upper right corner depicts the 3-phase motor-generator, transformer, rectifier, and DCDC converter that is providing power onto the PCC. To the left of the PCC are all of the components making up the actively controlled HESM. The two converters in the upper left corner allow power to flow to the left and charge the batteries when there is excess power from the generator. When the batteries need to supply power, the buck converter below allows power to flow to the right, discharging the batteries and providing power to the load. Control of those converters is achieved using a National Instruments (NI) CompactRIO platform deploying a fuzzy logic controller. A $3.5 \mathrm{~kW}$ AC/DC Chroma programmable load is used to load the PCC.

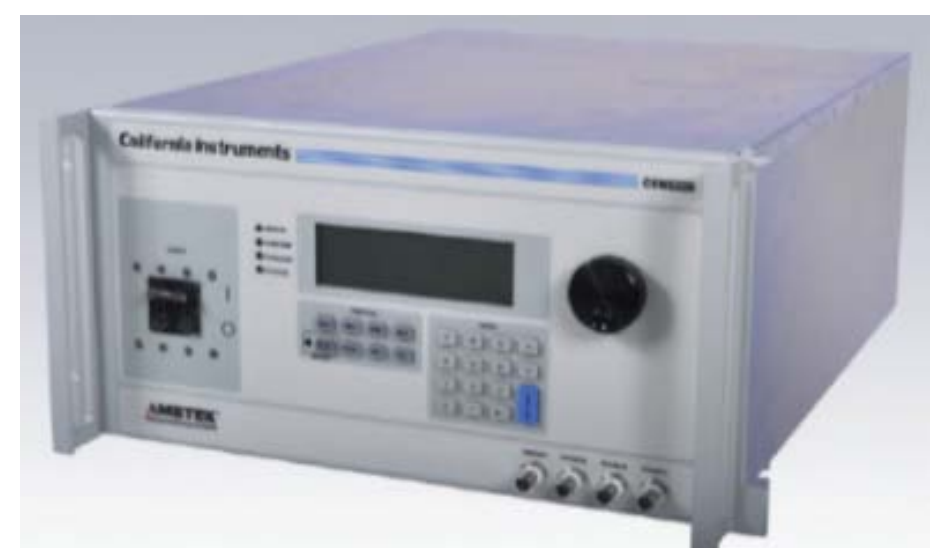

Figure 7. CSW 5550 3-phase power supply. Rated at 5 kVA with per-phase analog control.

Figure 9 depicts the same setup, except in this case the motor-generator is replaced with the 3-phase power supply that is controlled by the OPAL-RT HIL real-time simulator. The red arrow represents the analog control being applied to the power supply and the green arrow represents the feedback signals used by OPAL-RT to update the model. An Ideal Transformer Model (ITM) interface algorithm was used in this work to interface the OPAL-RT to hardware. Figure 10 shows the physical assembly of the hardware. 


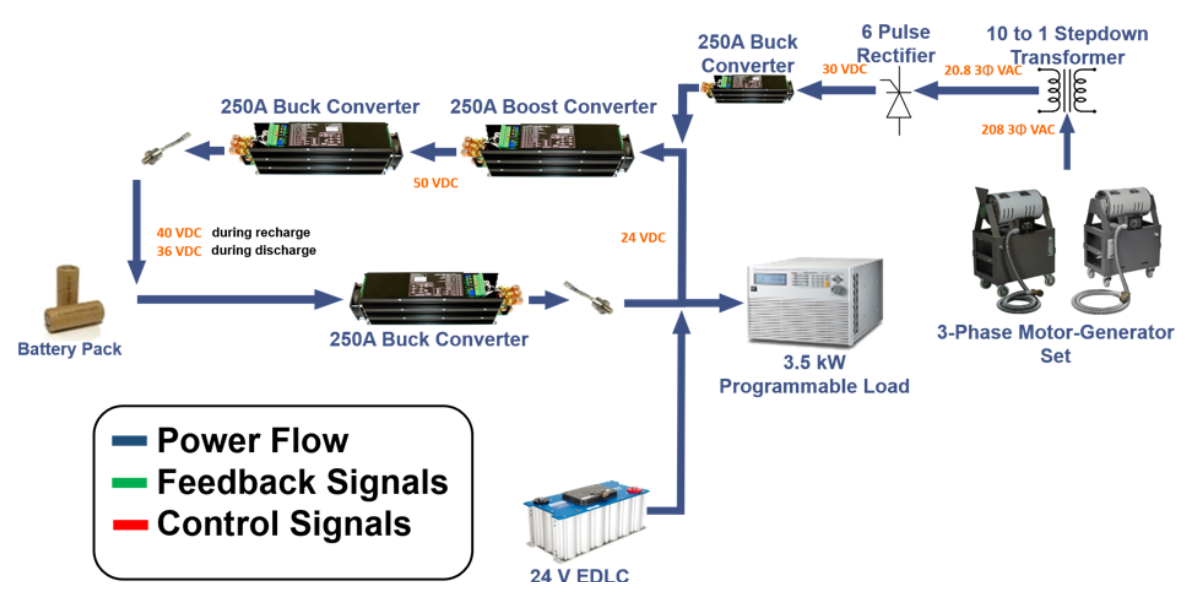

Figure 8. Block diagram of the hardware topology deploying a physical hardware motor-generator set.

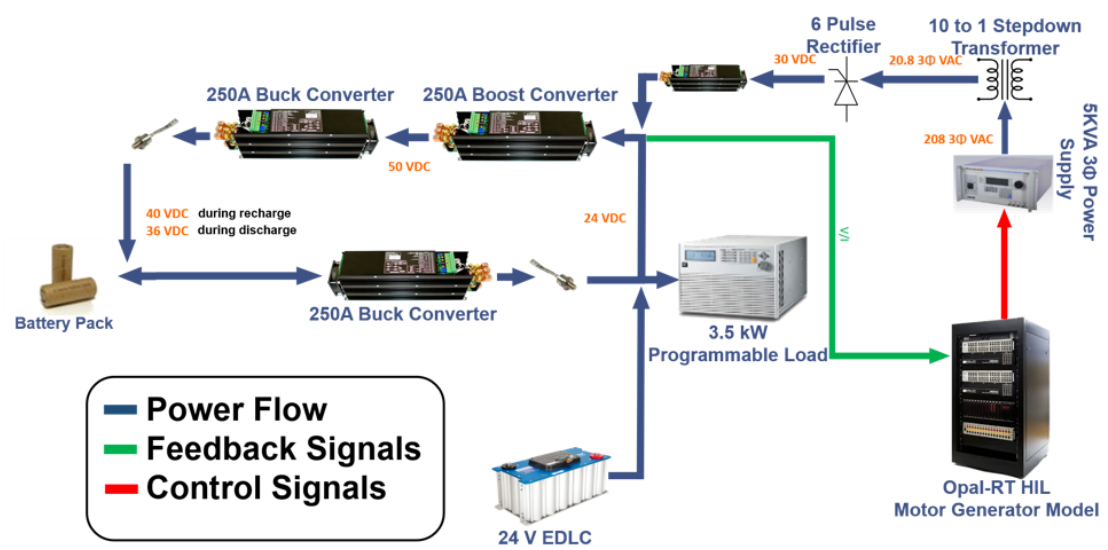

Figure 9. Block diagram of the hardware topology deploying a PHIL emulated AC generator.

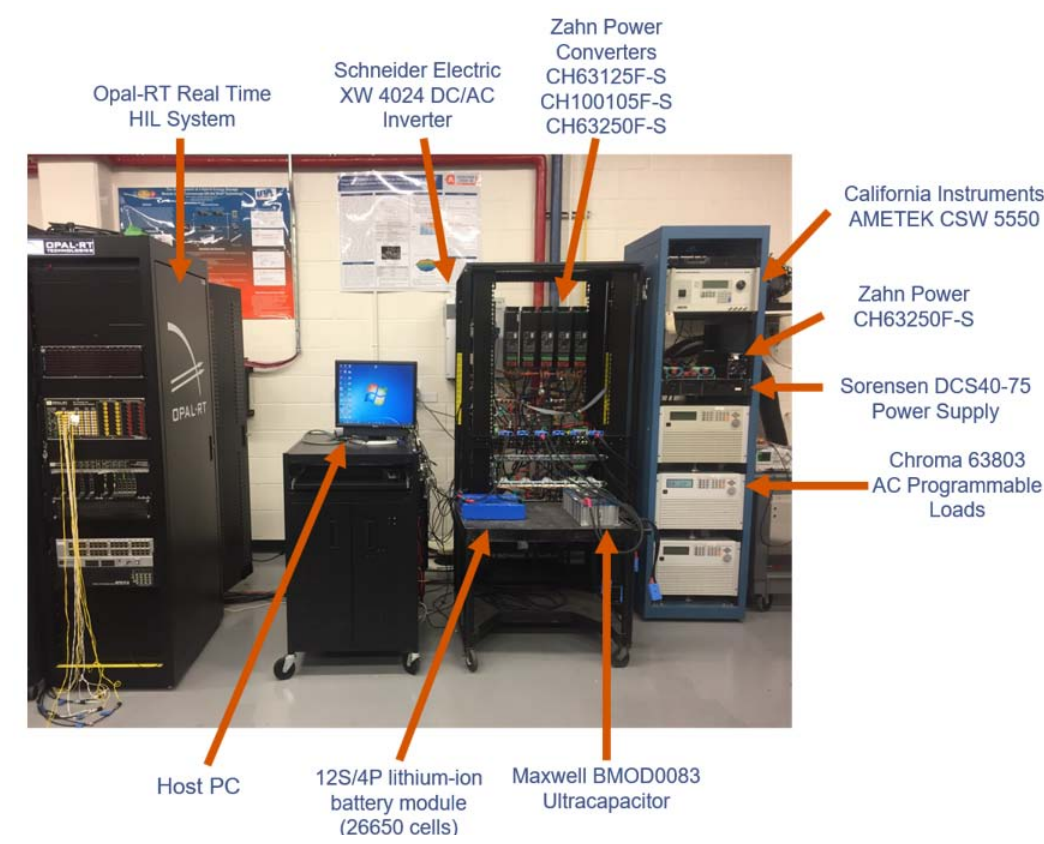

Figure 10. Picture of many components of the power system being studied. The physical motor-generator set is located behind the HESM. 


\section{Results}

In all the results presented here, the programmable load was setup to draw a constant current (CC) from the PCC in a transient manner. The profile executed turned the load on for 5 seconds, drawing 31 A CC, and then turned it off for 1 second before repeating four more times, five times total. Assuming 31 A and roughly $24 \mathrm{~V}$, the load was drawing roughly $750 \mathrm{~W}$ when on. In each series of experiments performed, two identical tests were performed. In one of the tests the actual motor-generator set was utilized while in the other the motor-generator set was emulated using PHIL. There are some discrepancies in the timing of some aspects of the waveforms. This is likely due to the National Instruments controller not operating with a real-time clock and serial communication delays to the electronic load, since each experiment must be performed independently.

\subsection{No buffering of the AC sources}

In this subsection, experiments were performed to obtain a comparison of the data observed when the physical motor-generator and the PHIL emulated generator were utilized, respectively, without the HESM providing any buffering. In other words, the transient load is supplied entirely by the motor-generator. Figure 11 shows the load current being drawn by the programmable load. In all of the plot legends, "MG" refers to the data collected with the physical motor-generator was used as the source and "HIL" refers to data collected when the AC source was emulated using HIL. As mentioned above, there is a slight, variable delay between the "MG" and "HIL" waveforms due to a delay in communication to the electronic load, as well as the software controlling the electronic load not operating on a real-time computer. In future work, these delays should be accounted for. The OPAL-RT could be used as a real-time computer to prevent time delay or dilation, and timing issues could be compensated for. The current sourced by each AC source in its respective experiment is plotted in Figure 12. This pulsed loading results almost $5 \mathrm{~V}$ of droop on the PCC DC bus as seen in Figure 13. These figures show the inefficiency resulting from pulsed loading without the HESM active, as well as poor power quality on the DC bus. As expected however, the emulated source matches the physical source waveforms well.

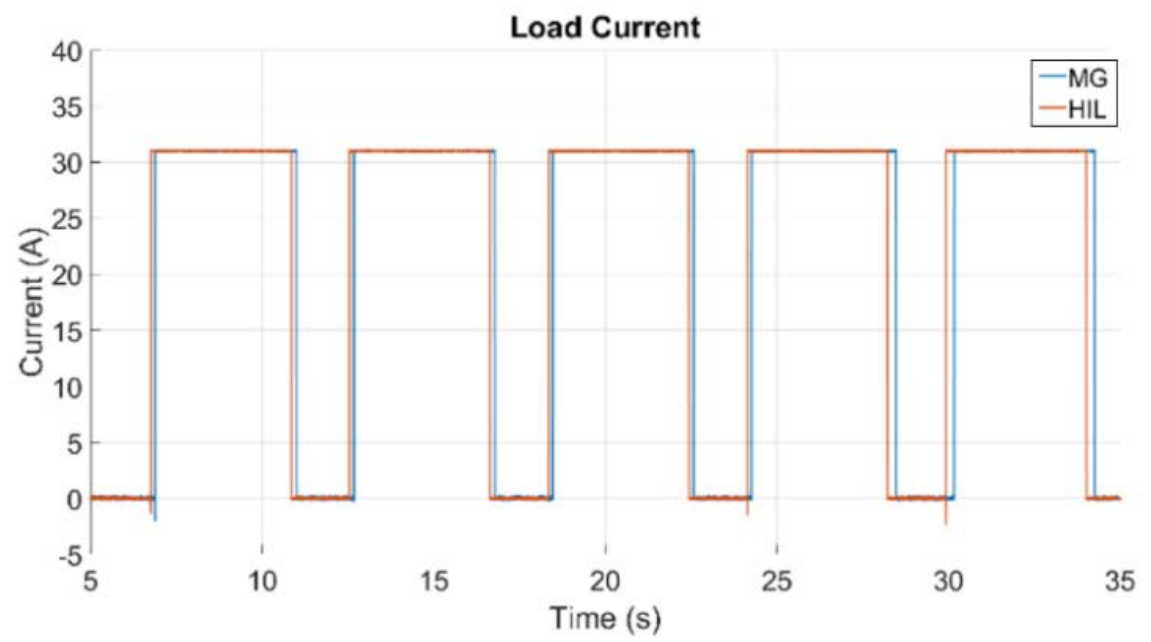

Figure 11. Current drawn by the programmable load during each respective experiment performed in which no HESM was used to buffer the AC source.

The AC voltages measured on Phase B of each source during its respective experiment are shown in Figure 14. Though the two voltage waveforms have slightly different magnitudes, closer inspection of the waveforms in Figure 15 shows that the variation in magnitude is largely due to harmonic distortion while the fundamental component of the phase voltages are very close. These are shortcomings of the model that must be studied in greater depth moving forward. Though the step-down transformer is present in both hardware and PHIL setups, further nonlinearities in the motor-generator magnetics, among other parasitic effects, could be the cause of the discrepancies. This effort is aiding in the understanding of how much deviation is acceptable for the HIL model to serve as a substitute for hardware, as complete modeling of system dynamics may not be feasible or practical in a given engineering application. 
Since the current waveforms change dramatically depending on the loading condition, the line current under no load is presented in Fig. 16 and the line current under full load is presented in Figure 17. Despite the difference in loading conditions, the emulated source matches the results obtained from physical motor-generator quite well. Despite the good agreement, there are variations in the degree of harmonic distortion that needs to be further modeled and studied. These results provide two valuable insights. First, they further validate earlier results obtained that show how utilization of an AC source alone to supply transient loads results in a degradation in both AC and DC power quality. Second, they suggest that the PHIL may be a valid limited substitute for AC generation sources that are not physically available for experimentation.

Rectified Current Supplied by the AC Source

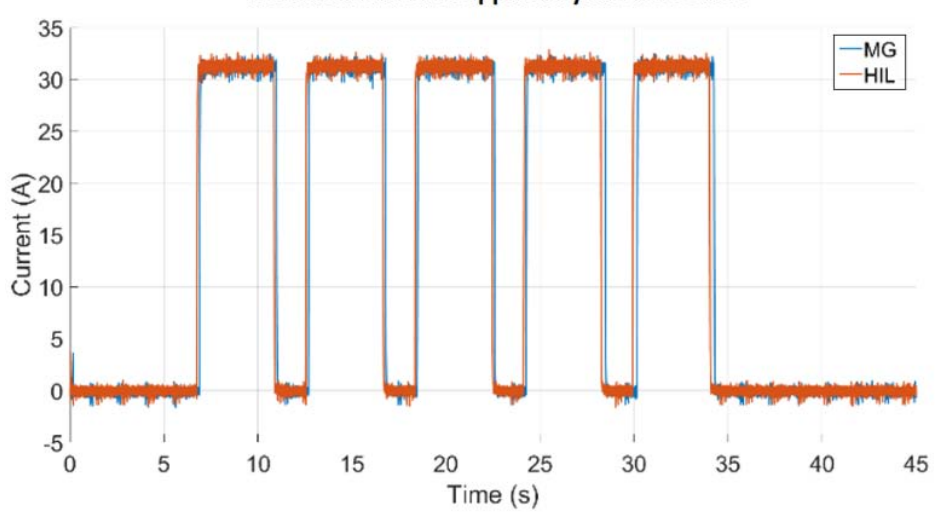

Figure 12. Rectified current supplied by the AC source during each respective experiment performed in which no HESM was used to buffer the AC source.

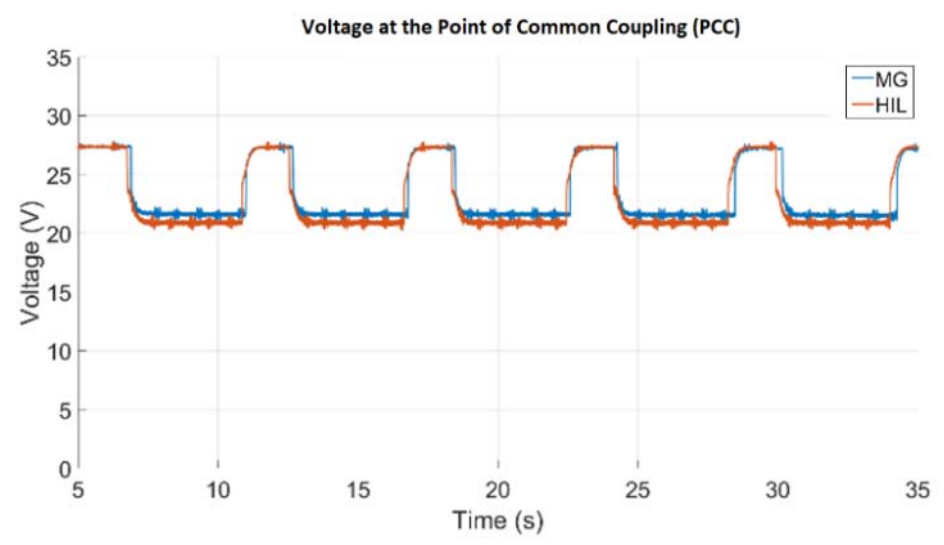

Figure 13. The PCC DC bus voltage showing deviation when the HESM is not used to buffer the AC source.

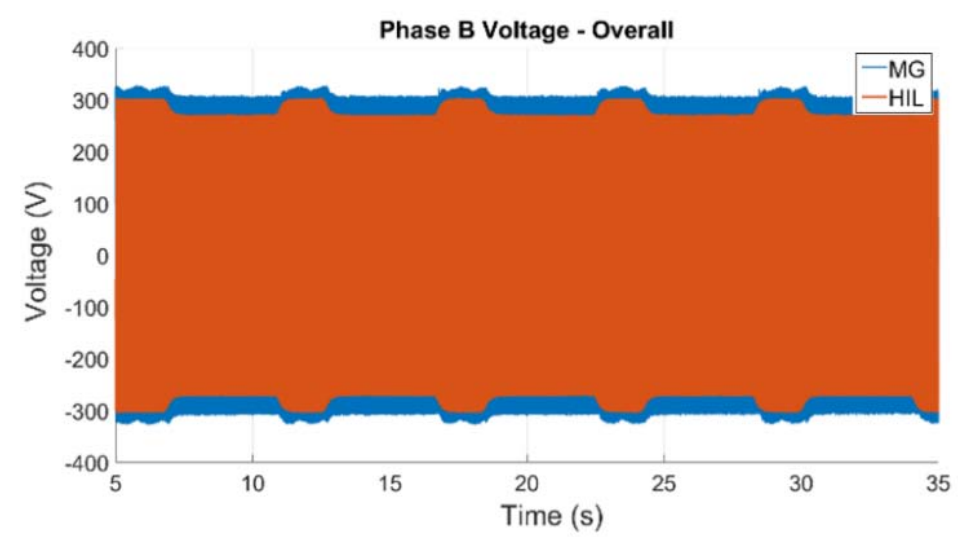

Figure 14. Phase voltage droops observed when the AC source is used to supply the full transient load current. 


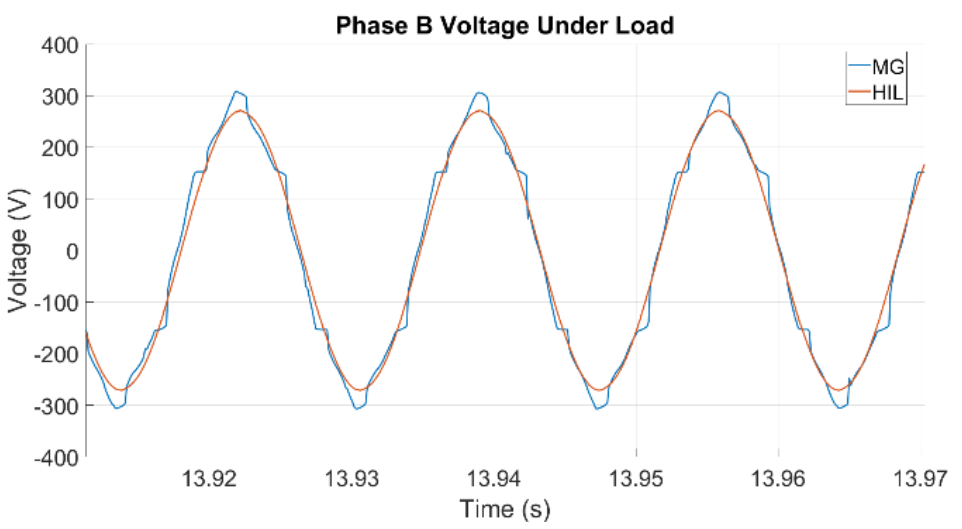

Figure 15. HIL source and motor-generator phase voltages are close despite harmonic distortion in the motor-generator.

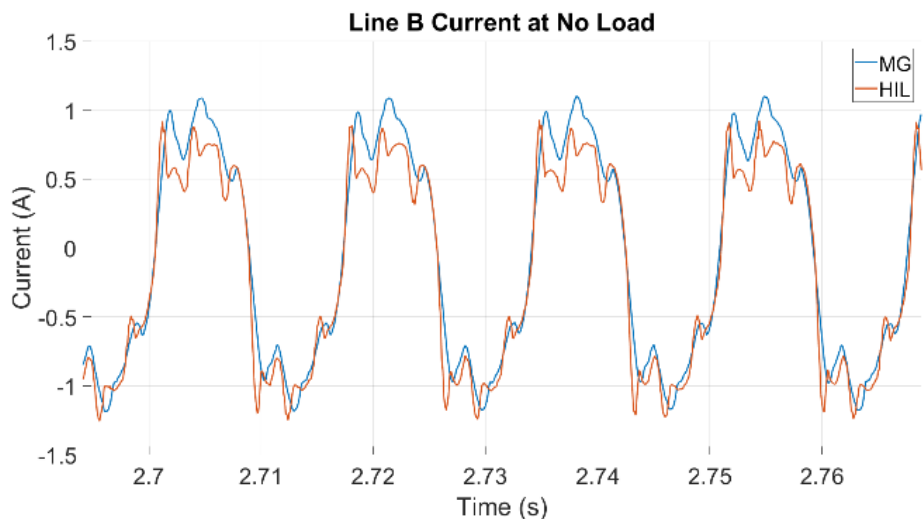

Figure 16. Line currents of each respective AC source during a 1 second rest period in experiments where no buffering supply is used. Fluctuations from the fundamental are caused by magnetization of the step-down transformer.

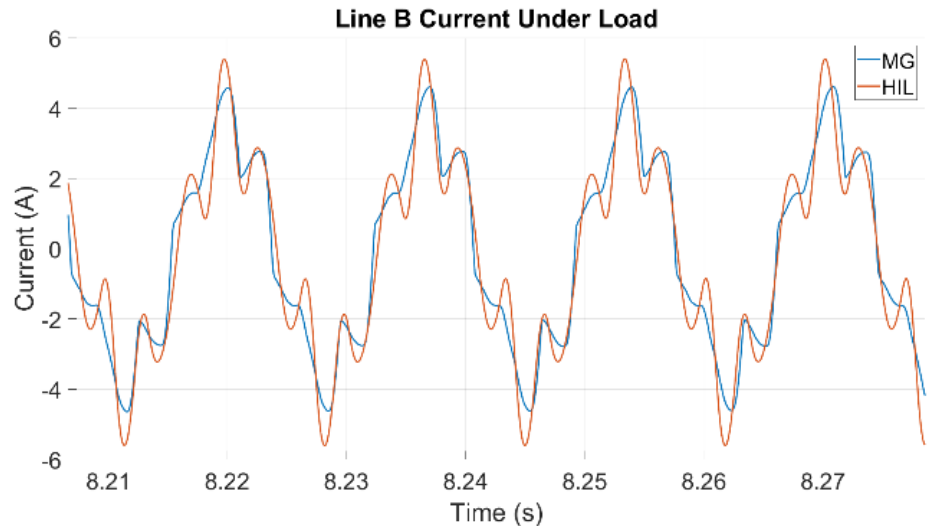

Figure 17. Line currents of each respective AC source during a 5 second load period in experiments where no buffering supply is used. Fluctuations from the fundamental are caused by magnetization of the step-down transformer.

\subsection{HESM actively buffering the sources}

In this subsection, results are shown in which the HESM is used to actively buffer the AC source. Figure 18 shows the same load profile being operated on the programmable load as that performed as in the previous series of experiments. Because the HESM is buffering the AC source, it is better loaded, supplying a nearly constant current to the PCC as shown in Figure 19. The HESM's batteries and capacitors providing a portion of the load current while it is on and continue to load the AC source when the load is off. This improves the stability of the PCC DC voltage bus considerably, shown in Figure 20, where deviations less than 1 V are observed. 


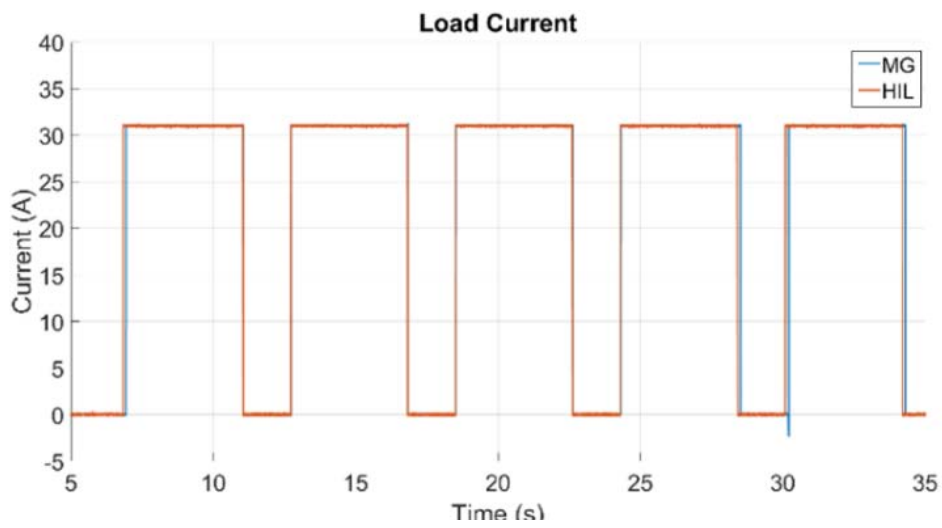

Figure 18. Current drawn by the programmable load during each respective experiment performed when the HESM was used to buffer the AC source.

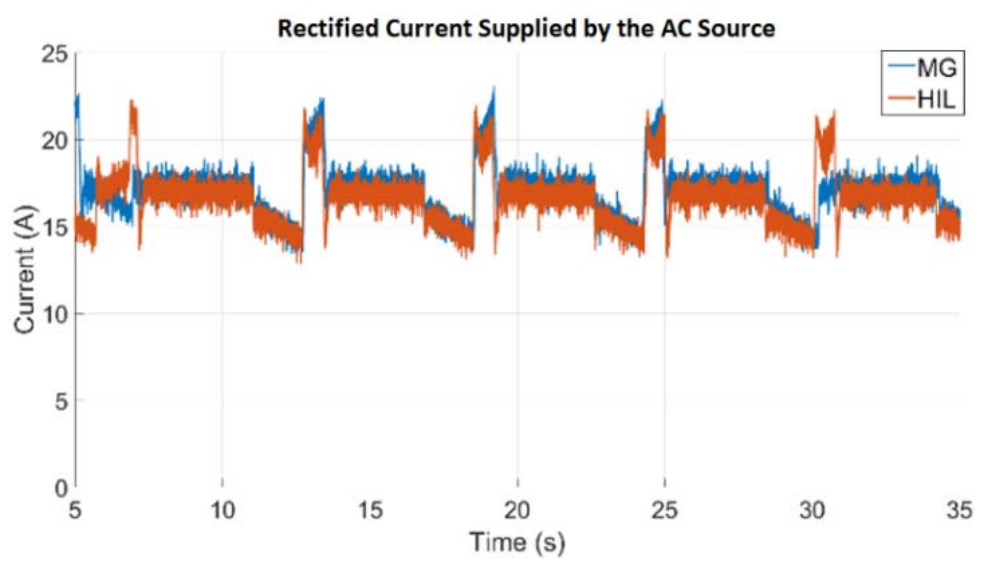

Figure 19. Rectified current supplied by the AC source during each respective experiment performed when the HESM was used to buffer the AC source.

Since the current sourced by the generator is largely consistent, the voltage droop in the phase voltages remains quite stable across the entire test, as shown in Figure 21, as compared to similar plots earlier in Figure 14 when no HESM was used. As was observed earlier in the unbuffered test there is harmonic distortion, but it is significantly reduced. Figure 22 and Figure 23 line currents in un-loaded and loaded conditions, respectively. The line current harmonics in the emulated source are very similar to the physical motor-generator's, however, improvements could be made with additional modeling.

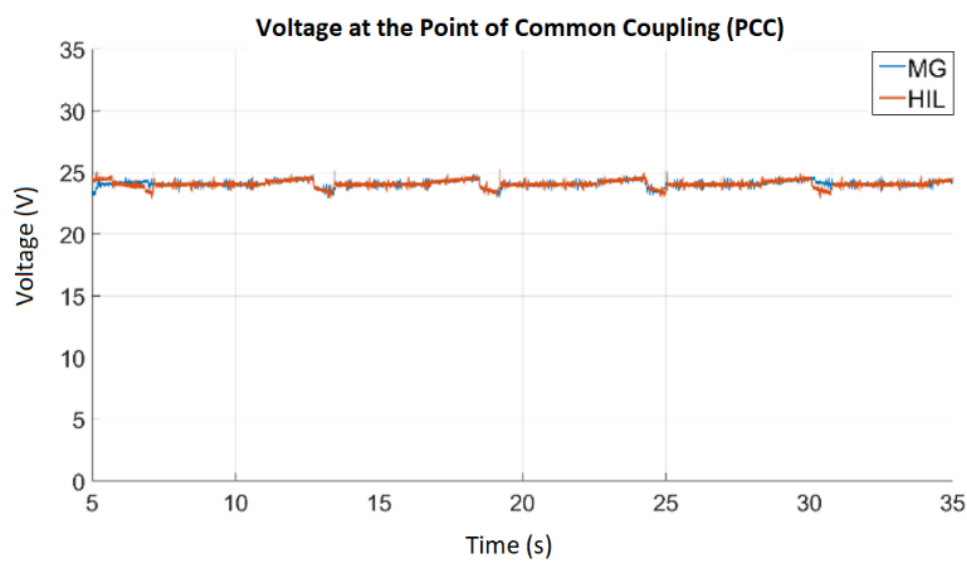

Figure 20. The PCC DC bus voltage showing much reduced deviation when the HESM is used to buffer the AC source. 


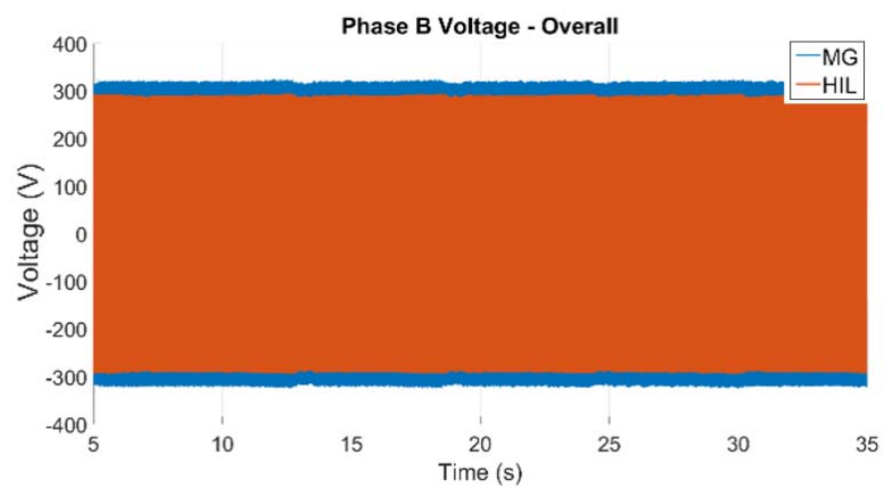

Figure 21. Phase voltage droops observed when the HESM is used to buffer the AC source.

The results of this series of experiments further confirms earlier results obtained that demonstrate the ability of an actively controlled HESM to improve the power quality of an AC source when they are used together to supply transient loads. The experiment shows that the data obtained from when using the PHIL emulated AC source very closely matches the data collected when a physical motor-generator set was used. There are areas for improvement, but the proof-of-principle experiment has demonstrated the potential for using PHIL in future experiments like these.

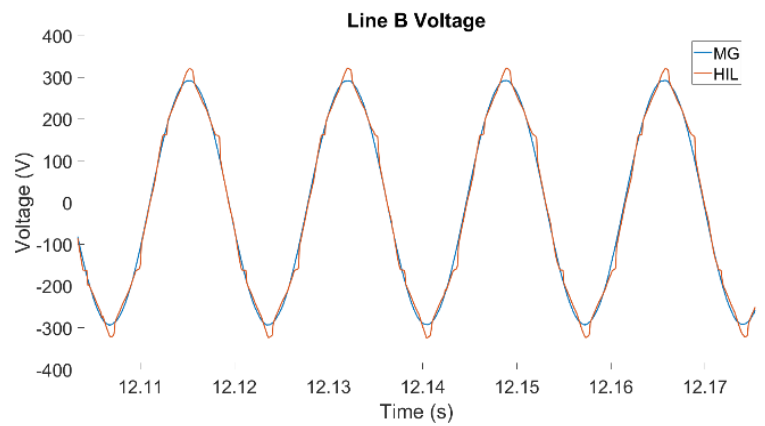

Figure 22. Line B voltage for each respective AC source during a 1 second rest period in experiments where the buffering supply is used. Fluctuations from the fundamental are caused by magnetization of the step-down transformer.

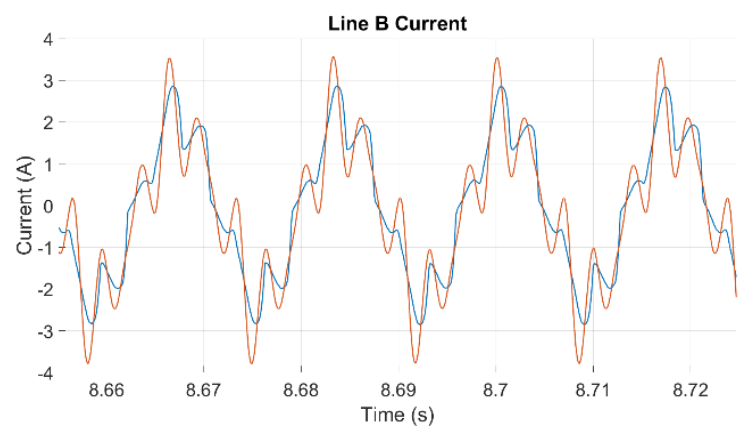

Figure 23. Line currents of each respective AC source during a 5 second load period in experiments where the buffering supply is used. Fluctuations from the fundamental are caused by magnetization of the step-down transformer.

\section{Conclusion}

The experiments and their results discussed here have shown a proof of concept for implementing PHIL emulated motor-generators for future work. The experiments have aimed to show the results that can be obtained when a simple Simulink motor-generator is used to model a generator possessed in house. The intent was to show what can be obtained with minimal model validation effort and to lay a foundation for future studies using motor-generator sets of higher relevance to shipboard and land-based power systems. The results have compared identical experiments performed with the hardware and the PHIL emulated hardware and have shown that while there are some 
minor discrepancies that need to be addressed with improved modeling techniques, the results are very similar. The questions still left to be answered concern how close is close enough when attempting to use this type of emulation to study power quality in larger power systems. It was shown that when good agreement between the hardware and simulation can be obtained without significant effort in modeling the nonlinear dynamics of the system that introduce harmonic distortion. The results indicate that the agreement can be improved considerably with more detailed motor-generator modeling and that is planned for future work with more relevant motor-generator set. Parameters such as power quality, voltage droop, and frequency deviation need to be studied on more relevant motor generator systems. The integration of additional hardware into the OPAL-RT model could show the ability to further remove additional physical components, such as the transformer and rectifier as well. This way, entire power systems can be modeled and deployed as a black box power system for interaction with other physical components.

\section{Acknowledgment}

The authors would like thank ONR for their continued support. Any opinions, findings, and conclusions or recommendations expressed in this publication are those of the authors and do not necessarily reflect the views of the US Office of Naval Research.

\section{Nomenclature}

PPEL—Pulsed Power and Energy Laboratory, HIL—Hardwarein the Loop, HESM-Hybrid Energy Storage Module, EDLCs_Electric Double Layer Capacitor, COTS—Commercial off the Shelf, PHIL—Power Hardware in the Loop, PCC—Point of Common Coupling, CC—Constant Current

\section{References}

[1] I. J. Cohen, D. A. Wetz, B. J. McRee, Q. Dong, and J. M. Heinzel. (2017). "Fuzzy logic control of a hybrid energy storage module for use as a high rate prime power supply,” IEEE Transactions on Dielectrics and Electrical Insulation, vol. 24, no. 6, pp. 3887-3893, Dec. 2017.

[2] I. J. Cohen, J. P. Kelley, D. A. Wetz, and J. Heinzel. (2014). "Evaluation of a Hybrid Energy Storage Module for Pulsed Power Applications,” IEEE Transactions on Plasma Science, vol. 42, no. 10, pp. 2948-2955, Oct. 2014.

[3] I. J. Cohen, C. S. Westenhover, D. A. Wetz, J. M. Heinzel, and Q. Dong. (2015). "Evaluation of an actively controlled battery-capacitor hybrid energy storage module (HESM) for use in driving pulsed power applications,” 2015 IEEE Pulsed Power Conference (PPC), Austin, TX, pp. 1-5, 2015.

[4] I. J. Cohen, D. A. Wetz, C. Storm, and J. Heinzel. (2014). "Impact of a Hybrid Energy Storage Module on Power Quality of a Fossil Fuel Generator,” Proceedings of the 2014 American Society of Naval Engineers Electric Machines Technology Symposium (EMTS), Philadelphia, PA, 2014.

[5] I. J. Cohen. (2016). “The Design and Control of a Battery-Supercapacitor Hybrid Energy Storage Module for Naval Applications,” The University of Texas at Arlington, Arlington, TX, May 2016.

[6] Marc Langevin, eng., Ph.D. Marc Soullière, tech. Jean Bèlanger, eng., "A Real-Time Regulator, Turbine and Alternator Test Bench for Ensuring Generators Under Test Contribute to Whole System Stability,” OPAL-RT Technologies.

[7] W. Ren, M. Steurer, and S. Woodruff. (2005). "Progress and challenges in real time hardware-in-the loop simulations of integrated ship power systems,” IEEE Power Engineering Society General Meeting, vol. 1, pp. 534-537, 2005.

[8] Y. Huo, G. Gruosso, and L. Piegari. (2017). "Power hardware in the loop simulator of photovoltaic plant for smart grid integration analysis,” 2017 IEEE International Conference on Environment and Electrical Engineering and 2017 IEEE Industrial and Commercial Power Systems Europe, Milan, pp. 1-5, 2017.

[9] M. Andrus, H. Ravindra, J. Hauer, M. Steurer, M. Bosworth, and R. Soman. (2015). "PHIL implementation of a MVDC fault management test bed for ship power systems based on megawatt-scale modular multilevel converters,” 2015 IEEE Electric Ship Technologies Symposium, Alexandria, VA, pp. 337-342, 2015.

[10] A. L. Gao, R. A. Dougal, and S. Liu. (2005). "Power Enhancement of an Actively Controlled Battery/Ultracapacitor Hybrid," IEEE Trans. Power Electronics, vol. 20, no. 1, pp. 236-243, 2005. 